\title{
Synthesis and deposition of Silver nanostructures on the silica microsphere by a laser-assisted photochemical method for SERS applications
}

\author{
Thanh Binh Pham, ${ }^{1,2}$ Thuy Van Nguyen, ${ }^{1}$ Thi Hong Cam Hoang, ${ }^{3}$ Huy Bui, ${ }^{1,2}$ Thanh Son Pham, ${ }^{1}$ \\ Van Phu Nguyen, ${ }^{4}$ and Van Hoi Pham ${ }^{1,2 *}$ \\ ${ }^{1}$ Institute of Materials Science, Vietnam Academy of Science and Technology, 18 Hoang Quoc Viet Road, Hanoi \\ 100000 , Vietnam \\ ${ }^{2}$ Graduate University of Science and Technology, Vietnam Academy of Science and Technology, 18 Hoang Quoc \\ Viet Road, Hanoi 100000, Vietnam \\ ${ }^{3}$ University of Science and Technology in Hanoi, 18 Hoang Quoc Viet Road, Hanoi 100000, Vietnam \\ ${ }^{4}$ Vinh University, 182 Le Duan Street, Vinh City, Nghe An, Vietnam
}

Received October 25, 2020; accepted November 25, 2020; published December 14, 2020

\begin{abstract}
The homogeneous distribution of nano-metallic structures on the surface-enhanced Raman (SERS) substrates plays an important factor for high-sensitive Raman scattering measurement. In this paper, we present a low-cost laser-assisted photochemical method for making a SERS probe based on silver nanostructures, which are one-timely synthesized nano-silver structures, homogeneously deposited on silica microsphere surfaces. Achieved SERS-activity substrates with a homogeneous distribution of Ag-nanostructures are verified by a mapping technique on the surface of Ag-coated microsphere for the detection of low concentration of Rhodamine $6 \mathrm{G}$ in aqueous solutions in a range of $10^{-4}-10^{-9} \mathrm{M}$. The obtained results show that a SERS microsphere probe has a good repetition of SERS-activity in any space of sensing area, and large potential for application in a biochemical sensing technique.
\end{abstract}

Today, our modern society makes ever-increasing technological demands for applications in environment monitoring, food safety control and health care [1-3]. Among the technologies applied in biochemical sensing, the SERS has been shown a powerful spectroscopic technique for ultrasensitive and high selective biochemical detection due to its capability of providing "fingerprint" information on biochemical molecular structures. The enhancement mechanism of SERS has been explained by a highly local enhanced electromagnetic (EM) field on the noble metal surface due to the excitation of localized surface plasmon resonances and enhanced chemical interaction between adsorbate and noble metal nanoparticles [4-6]. Recently, in the literature, there have been several studies combining SERS activity with optical fibre [7-10]. Among the noble metals such as gold, silver, palladium, and platinum, which have normally been applied in SERS substrates; the Ag-nanostructure is considered to be one of the most excellent candidates for SERS applications due to its highly desired plasmonic properties, low-cost and simple fabrication/synthesis. SERS substrates based

\footnotetext{
*E-mail: hoipv@ims.vast.ac.vn
}

on Ag-nanostructure with various morphologies can be fabricated in many different ways, such as gamma irradiation, electron irradiation, electrochemical deposition, chemical vapour deposition (CVD), chemical synthesis, microwave processing, by hydrothermal or photochemical method [11]. But the homogenous distribution of Ag-nanostructure on the SERS substrate is a problem for sample preparation and repeating measurement results.

In this paper, we propose a novel low-cost laser-assisted photochemical technique, which will take one-timely synthesis and deposition of Ag-nanostructures on the silica microsphere surface, in comparison with a photochemical method using high power light emitting diodes (LED). The morphology and chemical composition of SERS probes are characterized by highresolution scanning electron microscope (HR-SEM) and Energy dispersive X-ray spectroscopy (EDX), respectively. The achieved SERS-activity substrates on nano-Ag coated silica microsphere probes demonstrate low concentration of Rhodamine $6 \mathrm{G}$ in aqueous solutions in a range of $10^{-4} \div 10^{-9} \mathrm{M}$. The homogeneous distribution of Ag-nanostructures on the microsphere surface was tested by a mapping technique on the SERS surface with a different Raman signal of $2 \%$.

The schematic diagram of the making process of SERS probes with Ag nanostructures on silica microspheres prepared in two steps is shown in Fig. 1. The silica microsphere probe on the multimode optical fibre with core/cladding diameters of 50/125 microns, supplied from Thorlabs (USA), was fabricated by wet chemical etching of the optical fibre end in the HF acid (30\%) solution for 30 minutes, and then a microsphere was formed by melting the etched fibre end with electrical arc between the couple of discharge electrodes, as shown in Fig. 1a. The Ag growth solution was prepared by mixing an optimal molar concentration of aqueous $\mathrm{AgNO}_{3}$ of $0.1 \mathrm{mM}$ (Fisher Scientific, UK), and $\mathrm{C}_{6} \mathrm{H}_{5} \mathrm{Na}_{3} \mathrm{O}_{7} .2 \mathrm{H}_{2} \mathrm{O}$ of 


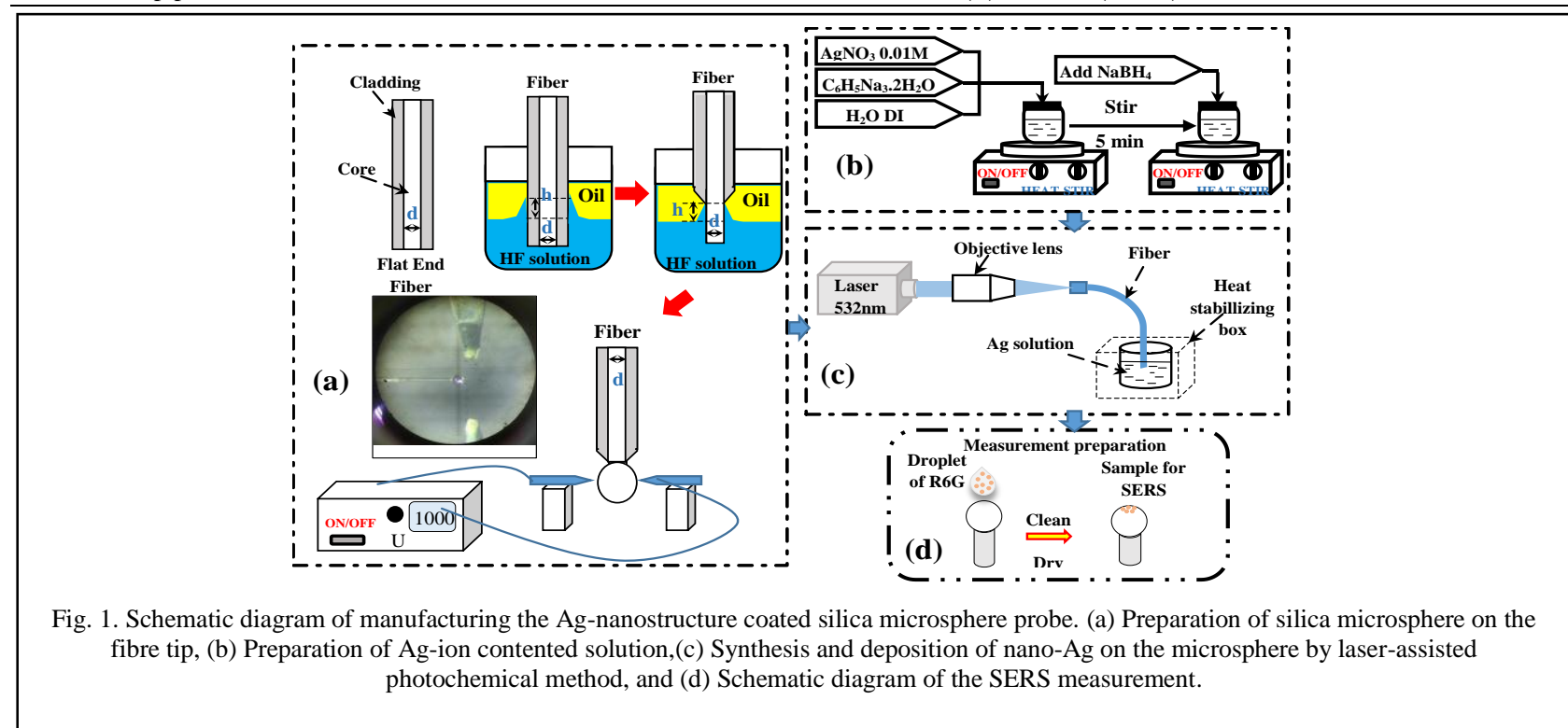

$3 \mathrm{mM}$ (Merck KGaA, Germany) in a volume ratio of 1:1 with rapid stirring for $5 \mathrm{~min}$, then a freshly prepared $0.02 \mathrm{mM} \mathrm{NaBH}_{4}$ solution (Kanto Chemical, Japan) was added by drop wise addition to the mixture under vigorous stirring for $15 \mathrm{~min}$, after which it was kept in the dark at room temperature, as shown in Fig. 1b. The growth and deposition of $\mathrm{Ag}$ nanostructures on surface microspheres were performed by a green laser with an emission wavelength of $532 \mathrm{~nm}$ and optical power of about $80 \mathrm{~W} / \mathrm{cm}^{2}$ (Laserlands, China), which propagated through the optical fibre to the silica microsphere attached at the end of this fibre (Fig. 1c). The growth and deposition of Ag nanostructures can be formed on a lightfocused area of the microsphere surface after illumination of few minutes. In our experiment, the reaction solution was contained in a box with a stable temperature at $3^{\circ} \div 5^{\circ} \mathrm{C}$ by using a Peltier thermoelectric cooler to avoid temperature effect on the growth of $\mathrm{Ag}$ nanostructures. After the silver growth and deposition process, the microsphere probe was taken out, carefully rinsed in deionized water and dried with a pure nitrogen gas stream. The schematic experimental setup of SERSactivity measurement using silica microsphere probes coated by Ag-nanostructures is shown in Fig.1d.

In order to investigate the performance of the SERSactivity of microsphere probes, we used ultralow concentrations of R6G solutions. An aqueous solution of R6G (Sigma-Aldrich) was diluted in deionized water to make solutions with different $\mathrm{R} 6 \mathrm{G}$ concentrations ranging from $10^{-4} \div 10^{-9} \mathrm{M}$. The droplets of $1 \mu \mathrm{L}$ of the R6G-diluted solutions were dropped on the SERS microsphere probes and naturally dried in air before SERS detection so that R6G molecules could be absorbed onto the $\mathrm{Ag}$ nanostructures. The Raman scattering measurements were performed at room temperature with a laser excitation wavelength of $532 \mathrm{~nm}$. The Ag nanostructure coated silica microsphere probe was fixed perpendicularly to the objective lens by the 5-stage holder (i.e., 3 stages for $\mathrm{XYZ}$ movement and 2 stages for angles change), and the exciting laser beam with a focused area of about $5 \mu \mathrm{m}$ in diameter and an incident light power of $3 \mathrm{~mW}$ has been irradiated on the top of microsphere. The typical spectrum acquisition time is $10 \mathrm{~s}$.

Figures $2 \mathrm{a}$ and $2 \mathrm{~b}$ show that the Ag-nanostructures are homogenously distributed on the surface of silica microsphere and the diameter area of Ag-nanostructure deposition is about $80 \mu \mathrm{m}$ when the core diameter of transmitting fibre is of 50 microns. Figure $2 \mathrm{c}$ displays the results of EDX of the Ag-nanostructures in the tiny region. The sharp peaks as Oxygen, Germanium $(\mathrm{Ge})$ and Silicon ( $\mathrm{Si}$ ) indicated the components of a typical optical fibre with Ge doped into an $\mathrm{SiO}_{2}$ substrate, and the sharp peak Ag definitively showed the growth of Agnanostructures deposited on the silica sphere. The nanoAg boulders of $100 \div 250 \mathrm{~nm}$ formed after a laser exposure time of 10 minutes, as shown in Fig. 2 d.

The SERS-activity of Ag-nanostructures was carried out with a diluted R6G solution using the Raman Microscope system (Horiba Scientific LabRAM HR Evolution). Figure 3 illustrates the SERS spectra of a R6G solution with various concentrations ranging from $10^{-4} \mathrm{M}$ to $10^{-9} \mathrm{M}$ dispersed onto Ag-nanostructure coated microsphere probes produced by the same synthesis process. It can be observed that the intensity of the SERS signal increases as the concentration of R6G increases. The Raman peak bands can be clearly observed in solutions with R6G concentrations down to $10^{-9} \mathrm{M}$. The strong Raman bands of R6G-adsorbed $\mathrm{Ag}$ nanostructures were clearly observed with the feature characteristic peaks at around $611.3 \mathrm{~cm}^{-1}, \quad 772.8 \mathrm{~cm}^{-1}, \quad 1181.9 \mathrm{~cm}^{-1}$, and $1308.4 \mathrm{~cm}^{-1}$, $1361.3 \mathrm{~cm}^{-1}, 1507.3 \mathrm{~cm}^{-1}, 1573.6 \mathrm{~cm}^{-1}$, and $1649.2 \mathrm{~cm}^{-1}$. To quantitatively estimate the $\mathrm{Ag}$ nanostructure-induced Raman enhancement, we calculated the enhance factor (EF) according to the formula [12]: 


$$
\mathrm{EF}=\frac{I_{\text {SERS }} \times C_{R}}{I_{R} \times C_{\text {SERS }}}
$$

where I $_{\text {SERS }}$ represents the enhanced Raman intensity of R6G adsorbed on an Ag-nanostructure functionalized microsphere probe and $I_{R}$ represents the normal Raman intensity (non-SERS) of R6G on the silica microsphere probe without Ag-nanostructure, $C_{S E R S}$ and $C_{R}$ are the concentration of R6G in the SERS spectrum and normal Raman spectrum, respectively.
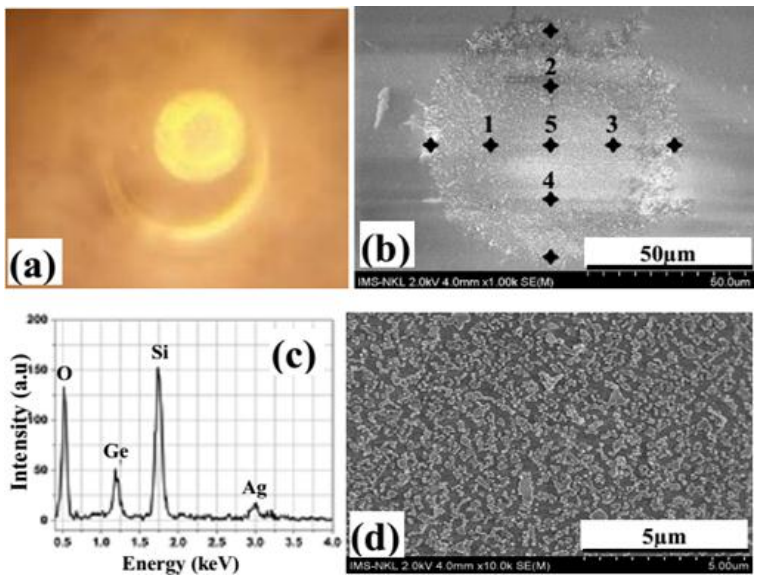

Fig. 2. (a) Optical photograph, and (b, d) the SEM images of Agnanostructure deposition area on the microsphere surface, and (c) EDX spectrum recorded on the Ag-deposited on the silica microsphere probe. The cross-makers in Fig. 2(b) present the mapping positions of SERS measurement.

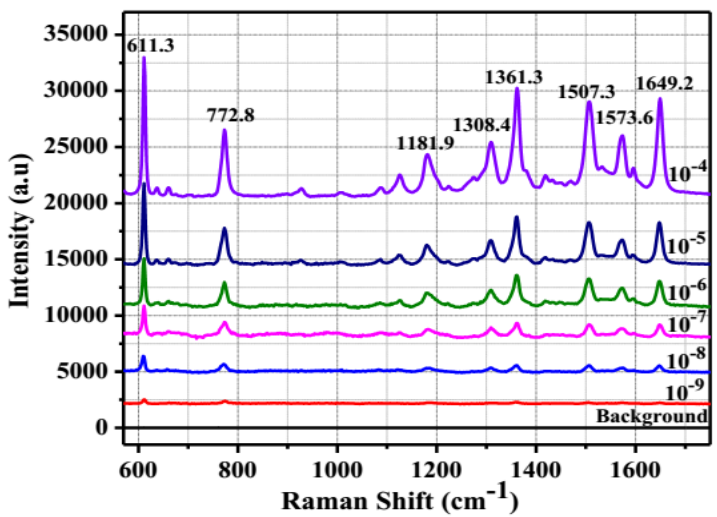

Fig. 3. SERS spectra of R6G solutions with concentrations ranging from $10^{-4} \mathrm{M}$ to $10^{-9} \mathrm{M}$

The Ag-nanostructure SERS exhibits excellent SERSactivity at the peak position of $1649.2 \mathrm{~cm}^{-1}$ and calculated EF value of $6.7 \times 10^{6}$. The mapping measurement of 5 positions on the nano-Ag coated silica microsphere probe indicated in Fig. 4 shows that SERS intensities of different positions at a Raman wavelength of $611.3 \mathrm{~cm}^{-1}$ for $10^{-4} \mathrm{M}$ of R6G varied $\pm 2 \%$, which demonstrates that the $\mathrm{Ag}$ nanostructures distributed homogenously on the surface of silica microsphere.

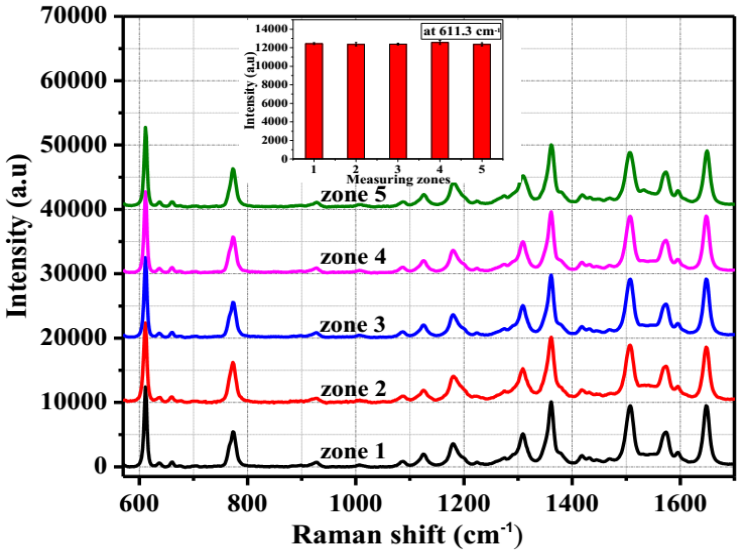

Fig. 4. Intensity of Raman spectra of R6G solutions with concentration of $10^{-4} \mathrm{M}$ measured by a mapping technique at 5 positions on a SERS substrate.

In summary, we have successfully grown and deposited Ag nanostructures on the surface of silica microsphere by a low-cost laser-assisted photochemical method, which can be used as a SERS-activity probe for biochemical molecular detection in a liquid environment.

The SERS-activity of an Ag nanostructure coated silica microsphere probe demonstrates its behaviour in detecting ultralow concentrations down to $10^{-9} \mathrm{M}$ of R6G with an enhance factor of $6.7 \times 10^{6}$. The $\mathrm{Ag}$ nanostructure coated silica microsphere probe has shown good repetition of SERS-activity in any space of the sensing area by homogenous distributions of $\mathrm{Ag}$ nanostructures and testing solutions dropped on the microsphere surface, which helps to develop a good quality SERS sensor for the detection of biochemical compounds in a liquid environment.

This work is financially supported by the NAFOSTED project No.103.03-2017.335 and a part of the VAST project under grant No.UDPTCN02/19-21.

\section{References}

[1] Y. Chen et al., J. Phys. D: Appl. Phys. 50, 02510 (2017)

[2] T.B. Pham, H. Bui, H.T. Le, V.H. Pham, Sensors 17, 0007 (2017).

[3] X. Wang, O.S. Wolfbeis, Anal. Chem. 88, 203 (2016).

[4] R. Wang, K. Kim, N. Choi, X. Wang, J. Lee, J.H. Joen, G. Rhie, J. Choo, Sens. Actuators B-Chem. 270, 72 (2018).

[5] H. Zhang et al., Anal. Let. 49, 2268 (2016).

[6] L. Chen, H. Yan, X. Xue, D. Jiang, Y. Cai, D. Liang, Y.M. Jung, X.X. Han, B. Zhao, Appl. Spectrosc. 71, 1543 (2017).

[7] A. Matikainen, T. Nuutinen, P. Vahimaa, S. Honkanen, Sci. Rep. 5, 8320 (2015).

[8] J. Zhang, S. Chen, T. Gong, X. Zhang, Y. Zhu, Plasm. 11, 743 (2016).

[9] W. Xu et al., Sensors 17, 467 (2017).

[10] K. Setoura, S. Ito, M. Yamada, H. Yamauchi, H. Miyasaka, J. Photochem. Photobio. A: Chem. 344, 168 (2017).

[11] K. Liu, Y. Bai, L. Zhang, Z. Yang, Q. Fan, H. Zheng, Y. Yin, C. Gao, Nano Lett. 16, 3675 (2016)

[12] Z. Huang, X. Lei, Y. Liu, Z. Wang, X. Wang, Z. Wang, Q. Mao, G. Meng, ACS Appl. Mater. Interfaces 7, 17247 (2015). 